\title{
Des cimetières lotis à Kisangani : exemple d'un débat occulte sur la gestion des espaces publics en RDC
}

\author{
Roger Ekongo Ndemba*
}

\begin{abstract}
Résumé
Dans les principales villes de la RDC, comme dans celles de la plupart des pays africains, les cimetières sont des espaces publics gérés par les municipalités. Jadis réservés aux morts et objet de respect, d'entretien régulier et de protection tous azimuts, ces sites sont de nos jours en proie à une gestion peu responsable de la part des pouvoirs publics. Les débats publics sur la question sont quasiment occultés. A Kisangani, la transformation de bon nombre de cimetières non désaffectés en des lieux d'habitation frise la profanation des ces sites. On y voit des vivants et des morts cohabiter côte à côte. Par-delà les problèmes de santé et d'hygiène publique, cette cohabitation pose en outre le problème de la révolution des croyances et des mentalités ainsi que celui de l'inefficacité des politiques publiques en matière d'urbanisation. Un débat public sur la question, grâce notamment à une société civile forte et dynamique et des pouvoirs publics responsabilisés, permettra de mettre fin à la banalisation du "sacré » et du « mystique " ainsi qu'à une gestion peu responsable de nos espaces publics.
\end{abstract}

\begin{abstract}
In the major cities of the DRC, as in those of most African countries, cemeteries are public spaces managed by the municipalities. Formerly spaces reserved for the dead and objects of respect, regular upkeep and protection of all kinds, these sites have now fallen prey to irresponsible management on the part of the public authorities. Public debates on the issue are virtually invisible. In Kisangani, the transformation of many cemeteries still in use into places of
\end{abstract}

\footnotetext{
* Université de Kisangani, Kisangani, RDC. Email : ekoroger2006@yahoo.fr
} 
residence comes close to a profanation of those sites. The living and the dead are side by side. Beyond the issues of health and public hygiene, this cohabitation also raises the issue of revolution in beliefs and mentalities as well as the issue of the inefficiency of public policy in terms of urban planning. A public debate on this issue, notably thanks to a strong and dynamic civil society and accountable public authorities, would make it possible to end the trivialization of the 'sacred' and the 'mystical' as well as the irresponsible management of our public spaces.

\section{Introduction}

L'accroissement spectaculaire et sans précédent de la population des villes en Afrique est devenu une question de société. Des débats intéressants ont été menés (Ela J. M. (1983), Streffler (1986), Le Bris (1987), dans la plupart des États en Afrique, lesquels étaient centrés singulièrement sur l'analyse des politiques publiques en matière de la gestion des espaces publics, en l'occurrence ceux que sont les villes.

L'essentiel de ces discussions a mis en évidence l'inadéquation entre les politiques publiques menées par les États et l'ampleur des besoins en matière d'occupation des espaces, surtout en milieu urbain.

Hardon et Listterth Waite, que cite Stren (1985:72) notent que l'action des gouvernements est loin d'approcher les niveaux de besoins; et les objectifs gouvernementaux ne doivent pas être confondus avec les réalisations concrètes. Dans presque tous les cas où les statistiques font apparaître des progrès dans la réalisation des objectifs officiels, notent-ils, le nombre d'unités effectivement construites reste néanmoins très éloigné des objectifs. De même l'action des gouvernements dans les domaines de la gestion et de la planification de l'espace public est presque toujours inadaptée et souvent mal orientée.

En plus de ces inadéquations des politiques publiques, il sied de souligner l'exode rural, facteur non moins négligeable dans la croissance urbaine. Le déplacement des populations des milieux ruraux, attirées par les centres urbains, marque le début de ce que Georges Balandier (1957:151) qualifie d'urbanisation hâtive et inquiétante.

Au-delà de l'étude de Régis Bertrand (2001), qui offre, sur les lieux d'inhumation stambouliotes,, une première synthèse axée tour à tour sur les questions du statut et de la gestion des cimetières et de leur place dans la ville, celles menées à Kisangani par Kangele Mwenda (1991) et Bitangi Mukombe (2002) se sont intéressées respectivement aux implications de la croissance urbaine sur la gestion des cimetières à Kisangani, et à la manière dont étaient organisés et gérés les cimetières dans la configuration urbaine de la ville de Kisangani. 
Par ailleurs, les écrits de Mavungu Konde (1988), Sadiki Mulindilwa (1996) et Ambolo Mosaki (2001) se sont limités à fustiger les conséquences de l'urbanisation anarchique de la ville de Kisangani. Les politiques publiques en matière de la gestion des espaces publics ne répondent pas toujours aux attentes des populations, lesquelles sont confrontées à un besoin pressant de logement. En effet, l'expression accrue de ce besoin débouche inévitablement sur une occupation anarchique des espaces.

Les cimetières, on le sait, sont parmi les espaces publics gérés généralement par la municipalité locale. Néanmoins, à Kisangani, l'ennui est que ceux-ci sont anarchiquement occupés. Bon nombre de cimetières non désaffectés sont en voie de disparition pour laisser la place aux constructions des maisons d'habitation. Cette réalité contraste avec les espaces à lotir dont regorge la ville.

Malgré l'ampleur du phénomène, la question semble, de nos jours, être occultée par les débats publics. En témoigne le débat autour des élections présidentielles et législatives de 2006. Il y a de fortes craintes que cette question soit de nouveau ignorée par les débats publics en cette période de fièvre électorale les élections générales sont prévues en RDCongo.

La crainte est d'autant plus grande lorsqu'on sait que la responsabilité directe de la gestion des cimetières de la ville ou de leurs entités respectives est dévolue aux autorités municipales.

- Pourquoi la question du lotissement anarchique des cimetières n’intéresse-t-elle pas le débat public malgré les dangers que ce phénomène représente ?

- Peut-on parler d'une certaine implication coupable des acteurs étatiques dans la gestion dangereuse de ces espaces publics?

- N'assistons-nous pas désormais à la suite de la profanation des ces lieux publics, à une banalisation du «sacré » face aux exigences de gestion des espaces publics et à la poussée de l'urbanisation anarchique de nos villes en Afrique?

La présente étude se propose de susciter le débat public autour de la question de lotissement des cimetières et d'attirer l'attention des autorités étatiques sur le danger que représente la gestion irresponsable des espaces publics. Partant de l'analyse des cas vécus dans la ville de Kisangani, en République Démocratique du Congo, cette communication s'efforce de relever les causes qui expliquent, dans le débat public, l'occultation de la question du lotissement anarchique des cimetières à Kisangani. 
Outre l'introduction et la conclusion, nous aborderons au premier chapitre la genèse des cimetières à Kisangani, au second chapitre, nous analyserons les problèmes de la gestion des cimetières à Kisangani et enfin, au troisième chapitre, nous allons nous appesantir sur quelques causes de l'occultation de la question du lotissement anarchique des cimetières des débats publics.

\section{Genèse et répartition de cimetières à Kisangani}

\section{Genèse des cimetières}

Pour mieux cerner les problèmes qui entourent la gestion de cimetières, il y a lieu d'interroger l'histoire, pour en savoir plus, sur la genèse de la création et de la gestion des cimetières dans la ville de Kisangani. Trois moments de l'histoire de la RDC donnent un éclairage sur l'évolution de la gestion des cimetières à Kisangani. Il s'agit des périodes pré-coloniale, coloniale et postcoloniale.

\section{La période pré-coloniale}

Ainsi que le note Kangele Mwenda (1991:35), la période ayant précédé la colonisation n'a pas connu une sorte de législation formelle en matière de la gestion de l'espace. Cette situation est consécutive à la création des villes par les colonisateurs, qui ont compris la nécessité d'établir les villes sur des espaces planifiés et mieux structurés. Néanmoins, il y a lieu de noter que les pratiques d'enterrement ont existé en tant que telles dans les sociétés indigènes avant l'érection de la ville de Kisangani. Selon les coutumes et les usages du milieu, les cimetières étaient confondus avec les maisons d'habitation dans certaines coutumes ; en revanche, dans d'autres, les cimetières étaient érigés loin des habitations. La terre, patrimoine de la communauté, était régie par la coutume et le chef du village en assurait la gestion. Ainsi, on pouvait trouver des cimetières dans chaque village.

\section{Pendant la colonisation}

En vue de mieux asseoir son entreprise, le colonisateur a mis sur pied une législation qui dépossédait les indigènes de leurs terres au profit de la couronne ou des compagnies à charte. La colonisation ayant occasionné l'urbanisation a, de ce fait, introduit un changement dans le domaine foncier dans la mesure où la terre, base et source de vie, appartenant à des communautés indigènes, a changé de propriété au profit des colonisateurs.

Selon Kangele Mwenda (1991:36-37), le Code civil belge, livre III, servait de réglementation en matière de gestion des cimetières. Au terme de ce Code, il est souligné que l'ordonnance du 4 mars 1908 du gouverneur général, modifiée par l'ordonnance n 336/j du 29 octobre 1947, réglemente le domaine de la création, d'inhumation et de réglementation des cimetières, 
en ville ou dans les villages. Cette loi précise en outre la nécessité d'établir un cimetière pour chaque groupement humain, de procéder aux inhumations uniquement dans celui-ci et de respecter la réglementation y afférente.

Un peu plus tôt précise Kangele Mwenda (1991:37), c'est-à-dire en 1902, la ville de Kisangani disposait déjà de quelques cimetières, après celui qualifié de plus ancien, à savoir le cimetière des prisonniers créé le 25 septembre 1895. En 1902, les trois cimetières suivants sont créés: l'ancien cimetière de Lubunga sur la rive gauche du fleuve Congo, le cimetière de Kabondo et le cimetière Belge I. Par la suite, d'autres cimetières seront progressivement créés à travers les différentes communes selon que le besoin se faisait sentir. Ainsi furent créés d'autres cimetières, notamment dans les communes de Makiso, en 1923, de Kisangani, en 1955, et de Lubunga, en 1957.

Par ailleurs, la saturation du cimetière Belge I avait conduit à ce que les dépouilles soient chaque fois transportées de Makiso vers la commune de Kabondo, sur la sixième transversale. En 1958, fut ouvert le cimetière de la rive droite de la rivière Tshopo pour servir les populations de la commune Tshopo et ses environs.

Pendant la période post-coloniale

Ainsi que le souligne Kangele Mwenda (1991:37), lorsque la gestion politicoadministrative passa aux mains des Congolais, de nouveaux sites funèbres furent créés. C’est ainsi que

- en 1966, fut créé le cimetière de la dixième avenue Tshopo, mais ayant son site dans l'entité de la commune Mangobo ;

- à Kabondo, fut créé en 1974 le cimetière de Kambakamba ;

- en 1991, fut aussi ouvert, à Mangobo, le cimetière de Ségama en vue de desservir séparément les communes de Mangobo et de Makiso.

Actuellement, la ville de Kisangani compte huit cimetières publics opérationnels, dont six sur la rive droite du fleuve et deux sur la rive gauche, à Lubunga. Des communautés particulières telles que l'église catholique, la communauté musulmane et celle des Indiens vivant à Kisangani disposent de leurs propres lieux d'inhumation.

\section{Evolution démographique et répartition des cimetières à Kisangani}

Evolution démographique

En réalité, l'implantation des cimetières a évolué en fonction de l'évolution démographique de la ville de Kisangani. Nous présentons dans les lignes qui suivent, et à titre indicatif, l'évolution de la population de la ville de Kisangani de 2000 à 2004. 


\begin{tabular}{cccc}
\hline \multicolumn{1}{c}{ Années } & Hommes & Femmes & Total \\
\hline 2000 & 368,278 & 369,112 & 737,039 \\
2001 & 387,188 & 378,567 & 765,755 \\
2002 & 406,023 & 406,944 & 812,967 \\
2003 & 466,325 & 427,291 & 853,616 \\
2004 & 415,509 & 453,438 & 869,006 \\
\hline
\end{tabular}

Source : Hôtel de ville de Kisangani, Rapports annuels de 2000 à 2004.

Il ressort de ce tableau que pour les cinq années considérées, la population de la ville de Kisangani s'accroît d'année en année, ce qui pose de manière évidente des problèmes socioéconomiques tels que le chômage et le manque accru de logement. Ce dernier, conduisant à son tour à des constructions anarchiques, peut pousser les populations à construire même dans des sites réservés à la sépulture.

\section{Répartition des cimetières}

Selon la proximité qui fait que deux ou trois communes urbaines partagent en commun un site de cimetière, les cimetières de la ville de Kisangani sont repartis en cinq zones comme suit: la zone est, la zone du centre-nord, la zone du centre-ouest, la zone du sud et la zone de Lubunga, vers la rive gauche du fleuve.

La zone est

Cette zone est située dans la commune urbaine de Kabondo. Elle est desservie par le site opérationnel qu'est le cimetière de Kambakamba, créé en 1974. Selon le préposé aux travaux publics et à l'aménagement du territoire de la commune Kabondo, ce cimetière a une superficie de 1 kilomètre carré. Deux autres cimetières, dont l'un situé à la sixième avenue, sont saturés et ont été désaffectés. La décision de fermeture de ces deux cimetières a été rendue officielle par arrêté urbain $n^{\circ}$ 031/MV/Kis/2002 du 14 novembre 2002. L'autre cimetière, également saturé, se trouve entre les quartiers Tshopo et Bakusu. De nos jours, bien que saturés, ces deux cimetières sont envahis par des constructions anarchiques.

\section{La zone centre-nord}

Cette zone est située dans la commune urbaine de la Makiso. Celle-ci s'étend vers le nord-est et même vers l'ouest de la ville. Elle regorge des cimetières suivants : 
- Le cimetière des pionniers de la ville. Il est situé sur le boulevard du 30 juin, en face du bâtiment de la CADECO, en plein cœur de la ville. Il s'agit d'un site où étaient enterrés les colons blancs avant l'indépendance du pays. Ce site est presque en voie de disparation, suite aux constructions qui y sont érigées.

- Le cimetière sis sur l'avenue du colonel TSHASTHI, au croisement avec l'avenue Abbé Munyororo, juste en face du bâtiment administratif de l'Université de Kisangani. Ouvert en 1923, ce site est complètement saturé et a été déclaré fermé par arrêté urbain ${ }^{\circ}$ 01/028/MV/Kis/ 2002 du 14 octobre 2002. Malgré cette décision officielle, des autorisations sont accordées à certaines personnes pour y enterrer leurs morts.

- Le cimetière du point kilométrique 6 , sur l'ancienne route Buta qui, en principe, est domaine privé de l'église catholique. Déclaré fermé, il continue malheureusement à recevoir des morts.

- Le cimetière de Segama, dans sa partie nord-ouest de l'aéroport de Simi-Simi. Ce site est exploité par deux communes, celle de Mangobo et celle de Makiso.

- Le cimetière Belge I situé au Bloc Kitenge, sur la colline, qui surplombe les services vétérinaires et la Faculté de Psychologie et des Sciences de l'éducation de l'Université de Kisangani. Bien que fermé et désaffecté, ce site est complètement envahi par des constructions de maisons d'habitation. En témoigne, par exemple, le nouveau bloc qui a fait son apparition sur les cendres de cet ancien cimetière.

- Le cimetière de la Croix Rouge, où ont été enterrées des centaines de milliers de victimes créées par des affrontements successifs entre les armées étrangères du Rwanda et de l'Ouganda, soutenues chacune par des factions rebelles congolaises en mai et juin 2000. Ce cimetière est situé derrière la morgue de l’Hôpital général de référence de Kisangani. Notons que d'autres morts n'y sont pas enterrés. Toutefois, ce site est ceinturé par des maisons d'habitation.

\section{La zone centre-ouest}

Cette zone renferme les cimetières situés dans les communes de Tshopo et de Mangobo.Dans la commune de Tshopo, l'on trouve un grand cimetière situé sur la rive droite de la rivière, qui porte le même nom. Ouvert en 1958, c'était, en réalité, le cimetière central de la ville de Kisangani. Il symbolisait la fin de la discrimination dans l'inhumation des morts entre les Blancs et les Noirs pendant la colonisation. 
À signaler aussi le cimetière dit de la $10^{\mathrm{e}}$ avenue, situé sur la chaussée menant vers la commune Mangobo. En effet, ce fut un cimetière satellite, car, en 1964, les ex-gendarmes katangais qui assiégeaient le pont Tshopo empêchaient les enterrements sur l'autre rive. Cela a conduit les autorités de l'époque à ériger un cimetière à cet endroit. Actuellement, ce site est saturé et tend à disparaître au profit des maisons qui y sont construites.

Dans la commune de Mangobo, les inhumations se font dans le cimetière dit de Segama. Bien qu'étant opérationnel, ce cimetière est ceinturé par des occupations et des constructions de maisons à telle enseigne que son extension pose aujourd'hui problème.

La zone sud-est

La zone sud-est, qui se trouve dans la commune de Kisangani, dispose d'une série de cimetières opérationnels. Le grand site est situé au quartier Konga Konga. C'est l'un des plus anciens sites de la commune créé en 1955. Étant saturé, il a été déclaré fermé il y a plus de deux décennies. En dépit de la saturation, ce cimetière continue à recevoir des corps pour y être enterrés. Les autres cimetières sont localisés le long de la route Ituri jusqu'aux limites de la commune Kisangani, avec les territoires d'Ubundu et de Bafwasende.

La commune de Lubunga

La commune de Lubunga est séparée des autres communes par le fleuve Congo. Ainsi, de par sa position géographique, elle est en retrait par rapport aux autres communes et dispose de ses propres cimetières. On y compte deux grands sites: le cimetière de Losoko créé en 1957 et celui de Katanga créé en 1961. Le premier est le plus vaste et se trouve à plus d'un kilomètre de la cité, tandis que le second est séparé de la cité par la rivière qui porte son nom.

Dans le chapitre qui suit, nous tentons d'identifier les causes profondes qui sont à l'origine de la profanation des cimetières à Kisangani.

\section{Les causes de lotissement des cimetières à Kisangani}

Nous distinguons ici les causes liées à l'administration de celles liées au changement des mentalités de la population.

\section{Causes liées à l'administration}

Il convient de souligner que la gestion des cimetières revient à l'autorité municipale. C'est le Bourgmestre qui gère au quotidien ces sites. Dans sa mission, le Bourgmestre de la commune bénéficie de la collaboration des services techniques suivants : 
1. Le service du cadastre et titre foncier qui, juridiquement, a la responsabilité de la gestion des terres en RDC. Ainsi, il oriente les affectations des terres pour un usage tant public que privé.

2. Le service de l'urbanisme et de l'habitat a la charge de fixer le plan d'érection ou d'extension du cimetière au regard des normes urbanistiques en vigueur.

3. Le service des travaux publics et de l'aménagement du territoire qui s'occupe de la mise en exécution du plan, de l'entretien et de la garde du site.

4. Le service de l'état civil et population qui se charge de la livraison des permis d'inhumation, document indispensable pour pouvoir enterrer quelqu'un dans le cimetière.

Chacun de ces services est représenté dans la commune par des agents de la fonction publique qui y sont affectés. Il convient de noter ici que ces services devraient en principe fonctionner au moins avec trois agents par service. Il en va de même pour les quatre services mentionnés ci-dessus. On devrait donc compter en principe 72 agents pour les six communes.

Nos enquêtes ont révélé que pour toutes les communes de la ville de Kisangani, ces services techniques ne fonctionnent qu'avec 13 agents, dont la ventilation est présentée dans le tableau qui suit :

Ventilation par commune des agents affectés aux services de cimetières dans la ville de Kisangani :

\begin{tabular}{clc}
\hline $\mathrm{N}^{\circ}$ & Commune & Effectif des agents \\
\hline 1 & Mangobo & 3 \\
2 & Kabondo & 2 \\
3 & Makiso & 2 \\
4 & Tshopo & 2 \\
5 & Lubunga & 2 \\
6 & Kisangani & 2 \\
Total & & $\mathbf{1 3}$ \\
\hline
\end{tabular}

Source : Nos enquêtes sur terrain.

Ce tableau montre que le nombre des agents pour les six communes est réparti comme suit : 3 agents pour la commune de Mangobo, 2 pour la commune de Kabondo, 2 pour la commune de Kisangani, 2 pour la com- 
mune de Tshopo, 2 pour la commune de Makiso et 2 pour la commune de Lubunga.

Il y a lieu de souligner ici que ces effectifs réduits constituent un obstacle réel à une gestion responsable des cimetières. De même, le travail de gestion des cimetières semble être négligé par l’État congolais. Ceux qui sont affectés comme gardiens des cimetières ou comme fossoyeurs sont pour la plupart de vieilles personnes ou de vieux retraités de l'administration publique. Par rapport à ces effectifs réduits, il convient de s'interroger sur la rémunération desdits agents. Si l'on se réfère à l'indice barémique actuel, un agent de bureau de la fonction publique gagne mensuellement 22420 francs congolais, ce qui représente environ 38 dollars américains.

La rémunération de cet agent représente en réalité une valeur faible en termes de pouvoir d'achat. A titrer illustratif, pendant que cet agent touche un salaire de 22420 francs congolais, un pagne pour dame coûte 15 000FC, soit environ 25 dollars, et les frais de scolarité par enfant s'élève à 18 000FC, soit environ 30 dollars pour une année scolaire.

Ainsi donc, l'agent est incapable de se procurer un pagne pour son épouse, de payer les frais de scolarité à son enfant, etc.

Exposé à des besoins sociaux, l'agent sous-payé ne pourra pas résister à la tentation de vendre anarchiquement des terrains pour subvenir à ses multiples besoins. Ainsi, les agents de l'État clochardisés cèdent aux sollicitations des demandeurs de terres, à qui ils vendent des terrains situés aux alentours ou au sein même des cimetières. C'est dans ce contexte socioéconomique précaire dû, sans aucun doute, à la démission de l'État que les agents chargés de la gestion des cimetières se livrent de plus en plus à la profanation des tombes en les octroyant aux populations qui en font des lieux d'habitation.

\section{La profanation des cimetières de Kisangani, suite au changement des mentalités}

La plupart des cimetières de la ville, du fait de leur surexploitation, sont en voie de désaffectation. Cette mesure de désaffectation vise à protéger l'espace ou le site funèbre. Face à la croissance urbaine que connaît la ville de Kisangani, les cimetières sont envahis par des maisons d'habitation. Autrement dit, les espaces de cimetières sont tout simplement lotis anarchiquement. Ces opérations permettent aux agents de l'État qui y sont affectés de gagner un peu plus d'argent, comme souligné plus haut.

A titre d'illustration, le cimetière Belge I, qui surplombe la colline sur laquelle est érigé le bâtiment des services vétérinaires, créé en 1902, fut déclaré saturé depuis 1956. Après avoir servi pendant plus de 44 ans, ce site a continué à servir de lieu d'enterrement jusqu’à 1984. Selon la législation 
en vigueur, il devrait être mis en valeur ou loti pour un autre usage seulement en 2034, après 50 ans. Mais actuellement, ce cimetière a disparu au profit des maisons d'habitation. Le cimetière de la $10^{\mathrm{e}}$ avenue, qui, en réalité, se trouve dans la commune de Mangobo, est lui aussi en voie de disparition. En lieu et place des tombes fleurissent des maisons d'habitation.

Il convient de faire remarquer que ce phénomène de profanation des cimetières n'est pas seulement l'œuvre des agents responsables de titres fonciers qui ont la charge de lotir les terres, mais que cela implique aussi la population qui accepte de les acquérir comme endroits d'habitation. A ce niveau, les mentalités et les croyances ont sensiblement évolué.

De nos jours, de nombreux discours religieux remettent en cause le caractère "sacré » ou " mystique " des cimetières que véhiculaient les croyances du passé. Selon ces discours, les esprits des morts élisaient domicile dans les cimetières et ces endroits étaient des cadres propices pour des rencontres « mystiques ». Actuellement, ce discours semble être dépassé et d'aucuns ne croient plus au caractère «sacré » ou «mystique » des cimetières. On y observe des gens qui vivent et mènent leur vie normalement. L'apparition, dans les sites des cimetières de Kinshasa par exemple, des activités de petits commerces informels, voire des pratiques de déterrement des cadavres pour en extraire les bijoux et autres biens de valeurs avec lesquels le cadavre a été enseveli s’inscrivent dans cette logique de révolution des mentalités dans la conception de la mort. Le chapitre qui suit examine pourquoi le débat sur les cimetières est occulté.

\section{Lotissement des cimetières à Kisangani : débat occulte sur la gestion de l'espace public}

Ce point analyse les causes de l'occultation de la question du lotissement anarchique des cimetières dans les débats publics à Kisangani. Trois raisons parmi tant d'autres nous ont paru majeures, à savoir la précarité de la situation socioéconomique, l'absence d'une société civile forte et dynamique, le manque de sensibilisation par les pouvoirs publics du plan d'urbanisation de la ville.

\section{La crise socioéconomique}

Répondre à un besoin de logement décent constitue pour l’État une manière de satisfaire aux demandes sociales de la population. L’émergence des quartiers d'habitation spontanée dans les sites où reposent les morts est pratiquement l'expression de la crise socio-économique aiguë que traversent nos États africains. Elle est la conséquence directe de la négligence et du refus des dirigeants politiques qui ne peuvent offrir des possibilités de logements décents et acceptables aux populations urbaines devenues de plus 
en plus denses. C'est le phénomène que René Maximy (1984:145) qualifie de logement d'auto-construction, fruit d'une jonction des besoins et des désirs des populations en matière de logement.

Dans un climat de crise socioéconomique, la croissance urbaine devient incontrôlée et plus qu'inquiétante. Les populations en proie à la crise de logement se ruent sur des sites destinés aux morts pour y ériger des logis de fortune. Dans presque tous ces quartiers érigés sur des cimetières, il est rare de trouver des maisons construites en dur. En revanche, ce sont des taudis qui pullulent, expression d'une population pauvre, abandonnée à ellemême, qui cherche par elle-même à résoudre ses besoins en logement. Ainsi, préoccupée par sa situation socioéconomique précaire, la population ne se soucie ni du débat public sur les questions de lotissement des cimetières, ni de l'exigence d'un débat public sur les cimetières lotis.

\section{L'absence d'une société civile forte et dynamique}

Les organisations de la société civile sont devenues une réalité indéniable dans les sociétés africaines actuelles. Elles se sont même imposées comme une des composantes incontournables sur la scène nationale.

Le rôle reconnu à la société civile est de recueillir les besoins et les aspirations des populations pour les canaliser vers les décideurs politiques en vue de solutions éventuelles. Elle peut aussi mobiliser la population en vue de la défense de ses intérêts. Etant constituée de personnes mieux informées, elle peut aussi alerter la population sur les conséquences d'une mauvaise décision ou d'une action politique donnée. Les organisations de la société civile jouent donc un rôle fondamental en identifiant les besoins sociaux des populations et en les formulant sous diverses formes de demandes sociales (environnement, développement social, habitat, sécurité sociale, santé, protection de l'enfant et de la femme, etc.).

La pression que ces organisations de la société civile exercent sur les décideurs politiques s'accroît au fur et à mesure qu'elles accèdent à l'information et au processus normatif et décisionnel. Pour que cette pression produise des résultats escomptés, il faut des leaders sociaux qui connaissent réellement les problèmes et les besoins des populations et qu'ils soient en mesure de mieux les exprimer et de les formuler sous forme de demandes sociales.

La société civile de la ville de Kisangani, et même de la RDC en général, a montré ses insuffisances et ses faiblesses quant à son aptitude à canaliser les aspirations de la population. A maintes reprises, elle est sortie atomisée, divisée par l'incapacité de ses animateurs à respecter le contrat social ou le cahier des charges contenant les aspirations profondes de la population. Elle est, dans sa majorité, diminuée et fragilisée à cause de sa quête du pouvoir et de l'avoir, bradant ainsi les intérêts de la population qu'elle est censée défendre. 
L’absence d'une réelle volonté de formuler les opinions susceptibles de limiter ou de mettre fin à la profanation des tombes reflète, entre autres, l’incapacité des acteurs de la société civile de connaître les aspirations réelles de la population sur cette question. En effet, cela les rend incapables d'élaborer des stratégies pour faire de la question du lotissement anarchique des cimetières un sujet de débat public auprès de l'opinion et des décideurs politiques.

Au cours de nos enquêtes, nous avons identifié des maisons d'habitation appartenant à certains leaders de la société civile ou aux membres de leur famille dans les sites de cimetières. Du fait de la complicité de ceux-là mêmes qui sont censés porter tout haut les besoins et les problèmes de la population avec les gestionnaires des terres, le débat public sur les lotissements anarchiques des cimetières dans la vile de Kisangani est occulté.

\section{Le manque de sensibilisation par les pouvoirs publics du plan d'urbanisation de la ville}

Dans tout État digne de ce nom, ce sont les pouvoirs publics qui élaborent et définissent la politique de l'aménagement et de la gestion de l'espace public. L'aménagement des espaces publics est une question qui doit être intégrée dans une politique nationale ou provinciale d'aménagement. S’il faut des moyens ou des ressources conséquentes pour mettre en place des infrastructures et des équipements urbains, il en faut davantage pour sensibiliser et informer la population sur les différents plans d'urbanisation en cours d'exécution. Mais, au contraire, les lotissements dans les cimetières sont la conséquence d'un manque d'information adéquate à l'égard de la population sur les différents plans cadastraux et d'urbanisation de la ville. Cette ignorance est entretenue par les autorités politico-administratives et par les services techniques impliqués dans la gestion des terres.

En effet, face à cette carence d’information délibérément entretenue, les autorités publiques n’hésitent plus à vendre des espaces non lotis pour des constructions. Ainsi, des espaces verts, des terrains de jeux, des concessions réservées pour un usage public, même des cimetières sont lotis au profit des maisons d'habitation. A ce niveau, il est pratiquement difficile d'envisager un réel débat public sur le lotissement des cimetières, car ceux qui sont sensés y veiller sont eux-mêmes impliqués dans la mauvaise gestion de l'information relative aux plans de lotissement.

\section{Le changement dans la conception de la mort en milieu urbain congolais}

Dans les sociétés traditionnelles congolaises en particulier, et africaines en général, la mort ne signifiait pas la fin absolue de la vie, mais seulement un changement d'état. Le respect dû aux morts faisait partie des valeurs 
essentielles de la société. Ainsi, tout ce qui se rapportait aux morts faisait partie du sacré et faisait l'objet d'une grande considération (Thomas 1982:39). Loin d'être considérée comme une rupture, la mort était plutôt un changement de statut. Les morts communiaient avec les vivants et ceux-ci recouraient fréquemment aux morts pour d’éventuelles solutions aux problèmes auxquels ils étaient confrontés. C'est ce qui explique que les survivants allaient se recueillir aux cimetières pour invoquer les esprits des morts, étant convaincus que ceux-ci sont dotés de la force du pouvoir, ainsi que de celle du savoir. Les morts ainsi que les lieux où ils reposent relevaient du sacré (Abel Nsolo 2006:7).

Mais de nos jours, cette conception tend à s'effacer à la suite des doctrines professées surtout par les églises dites de réveil qui prêchent qu’ une personne morte ne représente que la cendre, et que dans les tombes, il n’y a pas de vie. Cette révolution des mentalités face à la conception de la mort a donc banalisé le rôle jadis mystique et sacré des tombes, et cela a eu un impact déterminant sur l'affluence des populations vers les tombes, non plus pour y invoquer les esprits de leurs ancêtres morts, mais plutôt pour s'octroyer des parcelles et ériger des logis.

\section{Conclusion}

Notre propos avait pour objet de susciter le débat public sur la question du lotissement des cimetières. Il cherchait aussi à attirer l'attention des autorités étatiques sur le danger que représente une gestion irresponsable des espaces publics. Le lotissement anarchique des cimetières à Kisangani s'effectue à un rythme assez inquiétant. Si l'on n'y prend garde, tous les sites où reposent les morts à Kisangani auront disparu d'ici la moitié de la décennie en cours.

Pour enrayer ce phénomène croissant des constructions de logements dans les cimetières, il incombe aux autorités publiques d'orienter et de canaliser les besoins en logement des populations. Elles doivent aider les auto-constructeurs à suivre le plan d'urbanisation établi par l'État, au lieu que ces derniers puissent construire en désordre. L'État doit aussi renouveler la politique de l'octroi des crédits immobiliers aux populations et réglementer le taux de loyer pour les maisons à location.

Au-delà de toutes ces considérations, il importe que l’État assume ses responsabilités en assurant un salaire décent aux agents chargés de la gestion des lotissements, ce qui mettra fin à la précarité de leurs conditions de vie et les exposera moins à la corruption et au bradage des sites réservés aux morts. Néanmoins, le maintien d'un débat accru et continu sur la question, notamment à travers les médias, dans nos cités, à l'école et dans les universités nous semble être l'un des remèdes efficaces pour alerter l'opinion 
publique sur le danger de ce phénomène et inciter les autorités publiques à améliorer la gestion des espaces publics à Kisangani.

Pour que ce débat public soit constant, il faut une amélioration progressive des conditions de vie des populations, une société civile forte et dynamique et une large information de la population par les autorités sur les différents plans cadastraux et d'urbanisation de la ville, ainsi que sur les conséquences néfastes qui découlent au plan sanitaire par rapport à l'occupation effective des sites des cimetières. L'on devra également éduquer nos populations au respect des valeurs traditionnelles africaines, notamment celles du respect non seulement aux morts, mais aussi du caractère sacré des lieux où reposent ces morts. Car n'oublions pas que ces mêmes cimetières vont aussi accueillir tous ceux qui sont appelés à mourir un jour.

\section{Bibliographie}

Abel, N.H., 2006, La politesse au deuil, Kinshasa, éditions Paulines.

Ambolo, M., 1998, De l'évolution de l'urbanisation de la ville de Kisangani (19902000), mémoire de licence en sociologie (inédit), FSSAP, UNIKIS.

Balandier, G., 1957, L'Afrique ambigüe, Paris, éd. Union générale d'éditions.

Bitangi, M., 2002, La gestion des cimetières dans la ville de Kisangani, mémoire de licence en sociologie (inédit), FSSAP, UNIKIS.

Ela, J.M., 1983, La ville en Afrique noire, Paris, Karthala.

Le Bris, E., 1987, Famille et résidence dans les villes africaines, Paris, L’Harmattan.

Kangele, M., B.,1991, La croissance urbaine et ses implications sur les cimetières à Kisangani, mémoire de licence en sociologie (inédit), FSSAP, UNIKIS.

Mavungu, K., 1998, Analyse de quelques problèmes liés à l'urbanisation de la ville de Kisangani, mémoire de licence en sociologie (inédit), FSSAP, UNIKIS.

Maximy, R., 1984, Kinshasa. Ville en suspens : dynamique de la Croissance et problèmes d'urbanisation. approche socio-politique, ed. ORSTON, Paris, 1984.

Régis, B., 2001, « Vatin Nicolas et Yerasimov Stéphane, Les cimetières dans la ville. Statut, choix et organisation des lieux d'inhumation dans Istambul intra muros », in Istamboul, Institut français d'études anatoliennes Georges Dumézil, Paris, Lib. d'Amérique et d'OrientA. Maisonneuve.

Sadiki, M., 1996, Évolution de la gestion de l'espace urbain à Kisangani, mémoire de licence en sociologie (inédit), FSSAP, UNIKIS.

Streffler, F., 1986, Village, ville et migration au Zaïre, Paris, L'Harmattan.

Strener, 1964, « L’État risque de la ville », in Politiques urbaines, Paris, Karthala. Thomas, L-V., 1982, La mort africaine, Paris, Payot. 
